\title{
Natriuretic peptide-guided versus clinically guided therapy for chronic heart failure: careful expert clinical management can remove need for biomarker-tailored dosing adjustment
}

\author{
Renato De Vecchis \\ Cardiology Unit, Presidio Sanitario Intermedio "Elena d'Aosta”, Napoli, Italy \\ Correspondence to: Renato De Vecchis, MD, FESC. Cardiology Unit, Presidio Sanitario Intermedio “Elena d'Aosta”, via Cagnazzi 29, 80137 Napoli, \\ Italy. Email: devecchis.erre@virgilio.it; r.de.vecchis@alice.it. \\ Provenance: This is an invited Editorial commissioned by the Section Editor Hai-Long Dai (Department of Cardiology, Yan'an Affiliated Hospital of \\ Kunming Medical University, Kunming, China). \\ Comment on: Felker GM, Anstrom KJ, Adams KF, et al. Effect of Natriuretic Peptide-Guided Therapy on Hospitalization or Cardiovascular Mortality \\ in High-Risk Patients With Heart Failure and Reduced Ejection Fraction: A Randomized Clinical Trial. JAMA 2017;318:713-20.
}

Submitted Sep 29, 2017. Accepted for publication Oct 01, 2017.

doi: $10.21037 /$ jtd.2017.10.26

View this article at: http://dx.doi.org/10.21037/jtd.2017.10.26

This editorial refers to a randomized controlled trial (RCT), namely the Guided Evidence Based Therapy Using Biomarker Intensified Treatment in Heart Failure (GUIDE-IT) study conducted by Felker and colleagues, whose results have been recently published (1).

\section{GUIDE-IT study: a synthetic overview}

In the above-mentioned RCT, the aim of the research has been to ascertain whether a strategy that includes periodic measurements of serum amino-terminal pro-B-type natriuretic peptide (NT-proBNP) in a number of outpatients with chronic heart failure (CHF) is really able to achieve substantial benefits in terms of extension of survival free from adverse events and reduction of hospitalizations compared to conventional CHF management program.

As conventional clinico-therapeutic approach the authors deemed a CHF management program where drug dosages were adjusted based on the daily measurements of body weight and daily urine output variations, as well as on the basis of the expert assessment of clinical congestion's symptoms and signs, such as the intensity of dyspnea and the extent of peripheral edema.

The GUIDE-IT study was prematurely discontinued for futility because data recorded after a 15-month follow-up, when 894 of the 1,100 programmed patients had been enrolled, showed that the outcomes were substantially comparable in the two groups, i.e., they revealed no significant differences between the two arms.

In fact, the primary endpoint, i.e., the composite of timeto-first heart failure (HF) hospitalization or cardiovascular mortality, occurred in 164 out of a total of 446 patients $(37 \%)$ in the biomarker-guided group and in 164 out of a total of 448 patients $(37 \%)$ in the conventional care group [adjusted hazard ratio (HR), 0.98; 95\% confidence interval (CI), 0.79-1.22; $\mathrm{P}=0.88$ ]. Moreover, cardiovascular mortality occurred in $12 \%$ of patients in the natriuretic peptide (NP)guided arm and $13 \%$ of patients in the usual care arm (HR: 0.94; 95\% CI: 0.65-1.37). None of the secondary endpoints such as all-cause mortality, HF hospitalizations, days alive out-of-hospital or adverse events were significantly different between the two groups. Moreover, at 12 months there were comparable reductions in the serum NT-proBNP, similar percentage of patients with NT-proBNP of $<1,000 \mathrm{pg} / \mathrm{mL}$, as well as substantial overlap of patients who had reached target dosing of HF medications in the NP-guided arm compared to the arm treated with a clinically guided regimen.

\section{Background: historical importance of the experimental attempts aiming at realizing drug dosing adjustments for HF patients, based on systematic serum NP measurements}

As it is known, NP-guided therapy has not received an 
endorsement as the recommended approach for HF management by the American and European societal guidelines $(2,3)$. However, the usefulness of at least one NP measurement at the hospital admission and one at the time of discharge after hospitalization for an episode of acute decompensated heart failure (ADHF) was recently underlined by some authors $(4,5)$ because an anomalous NP curve, i.e., lack of decrease or paradoxical increase in NT-proBNP or B-type natriuretic peptide (BNP) serum levels during hospital stay for ADHF would be helpful in identifying patients with a particularly high risk of haemodynamic and clinical congestion, likely to require new hospitalizations for possible HF clinical relapses in the course of follow-up.

In truth, even several previous studies had resulted in quite disappointing outcomes for the advocates of the NPguided approach (6-8).

Notably, it was also argued (9) that in all of the studies that failed (6-8) in demonstrating a superiority of NP-guided approach, this disappointing result was to be attributed not to the ineffectiveness of the NP-guided strategy, but rather to the failure to follow the programmed study design, for which the investigators should have been more persevering in order to finally reach the NP target value as originally planned. Nevertheless, these arguments could be easily countered by emphasizing that sometimes an overt intolerance to increases in diuretics and vasodilator drugs, proven by occurrence of symptomatic hypotension and prerenal hyperazotemia may have dissuaded the investigators from reaching the predefined target and might have prevented them from making further increases in the doses. Yet, in other cases, even a reduction in glomerular filtration rate (GFR), which is a frequent finding in HF, may have contributed to the persistence of elevated circulating levels of NP by impeding its clearance and/or by favoring the occurrence of hormone resistance.

In the past, it has also been argued (9) that the reason for the alleged superiority of NP-guided therapy, as affirmed by some studies $(9,10)$ but not confirmed by others $(6-8)$, would be that the sensitivity of serial NP determinations in the detection of a still subtle and initial haemodynamic congestion is considerably higher than that exhibited by clinical criteria, such as a rapid weight gain, reduction in urine output or exacerbation of dyspnea on exertion. In fact, according to advocates of NP-guided approach, expert clinical assessment would be able to detect a hemodynamic deterioration less readily and less timely with respect to the serial serum NP measurements (either BNP or NT-proBNP).

According to this interpretation, drug adjustments would be earlier and more appropriate with a NP-guided pharmacologic management, and it would therefore be possible to correct the hemodynamic congestion of the incipient cardiac decompensation at a preclinical stage, namely, before the hemodynamic deterioration has resulted in a serious worsening of the clinical picture.

All of these concepts, however, appear substantially contradicted by the results of the GUIDE-IT trial.

\section{GUIDE-IT study: main remarks}

As regards the GUIDE-IT study, it should be remarked that, as expected, the frequency of medical visits, as well as the frequency of pharmacological dose adjustments, were higher in the patients' arm treated with $\mathrm{NP}$ - guided therapy, for both loop diuretics and evidence-based medications.

Nevertheless, neither doses of guideline-directed medical therapy, nor the achieved NT-proBNP concentrations, nor clinical outcomes were significantly different between the two groups, namely the one with NP-guided versus the one with clinically guided therapy. In particular, similarity of the proportions of patients who reached the target value of $\leq 1,000 \mathrm{pg} / \mathrm{mL}$ of NT-proBNP (46\% in biomarker-guided group $v s .40 \%$ in usual care group) is to be regarded as an unusual feature. Indeed, in previous studies, although in both NP-guided and clinically guided groups the target level of serum NP was attained only in a limited percentage of patients, nevertheless a more pronounced NP reduction in the NP-guided arm compared to clinically guided arm was a typical differential trait.

Several potential reasons may explicate why the findings from this study differ from those of some of the previous studies. In my opinion, an important reason consists in the fact that in the GUIDE-IT study, the pharmacological management of patients was generally more careful and accurate than in previous trials centered on the same topic. Indeed, in this trial a relatively large number of outpatient visits have been recorded not only in the NP-guided arm, but also in the arm of usual care.

Notably, in this trial, in contrast to most of the previous trials, remarkable endeavors were made to guarantee that patients in the clinically guided arm received optimized guideline-directed pharmacotherapy. In other words, there was a medical behavior tending to demonstrate that substantial reductions in NT-proBNP levels can be attained 
even with only empirical adjustment of drug dosing.

Perhaps the most reliable explication for the outcomes detected in the GUIDE-IT study is that when clinical care complies with guidelines and is diligently programmed to answer the key issues, biomarkers do not make a difference. In other words, guideline-directed care, if it can be meticulously and carefully accomplished, is more efficacious and can result in outcomes similar to those yielded by NP-guided therapy.

The GUIDE-IT study has some points of contact with a previous study, the Swedish Intervention study - Guidelines and NT-proBNP AnaLysis in Heart Failure (SIGNAL-HF) (11) trial, described with more detail in a synthetic review of my research team (12).

In short, even in the SIGNAL HF, dating from 2010, it is highlighted that NT-proBNP-guided treatment appears not to entail important improvements in clinical outcomes in patients with $\mathrm{CHF}$ in primary care above and beyond what could be achieved by education and structured $\mathrm{CHF}$ treatment according to guidelines.

\section{Conclusions}

The GUIDE-IT study is a further contribution to be added to several other previous studies that failed to document significant clinical improvement in terms of mortality and morbidity using a NP-guided strategy for CHF patients. Nevertheless, I believe that further larger and well-conducted trials addressing the unresolved issues of NP-guided therapy could still be helpful in the future.

\section{Acknowledgements}

None.

\section{Footnote}

Conflicts of Interest: The author has no conflicts of interest to declare.

\section{References}

1. Felker GM, Anstrom KJ, Adams KF, et al. Effect of Natriuretic Peptide-Guided Therapy on Hospitalization or Cardiovascular Mortality in High-Risk Patients With Heart Failure and Reduced Ejection Fraction: A Randomized Clinical Trial. JAMA 2017;318:713-20.

2. Yancy CW, Jessup M, Bozkurt B, et al. 2013 ACCF/AHA guideline for the management of heart failure: a report of the American College of Cardiology Foundation/American Heart Association Task Force on practice guidelines. Circulation 2013;128:e240-327.

3. Ponikowski P, Voors AA, Anker SD, et al. 2016 ESC Guidelines for the diagnosis and treatment of acute and chronic heart failure: The Task Force for the diagnosis and treatment of acute and chronic heart failure of the European Society of Cardiology (ESC). Developed with the special contribution of the Heart Failure Association (HFA) of the ESC. Eur J Heart Fail 2016;18:891-975.

4. Beltrami M, Palazzuoli A, Ruocco G, et al. The predictive value of plasma biomarkers in discharged heart failure patients: the role of plasma BNP. Minerva Cardioangiol 2016;64:147-56.

5. De Vecchis R, Ariano C, Baldi C. An Admission-toDischarge BNP Increase Is a Predictor of Six-Month All-Cause Death in ADHF Patients: Inferences from Multivariate Analysis Including Admission BNP and Various Clinical Measures of Congestion. J Clin Med 2016;5. pii: E99.

6. Lainchbury JG, Troughton RW, Strangman KM, et al. N-terminal pro-B-type natriuretic peptide-guided treatment for chronic heart failure: results from the BATTLESCARRED (NT-proBNP-Assisted Treatment To Lessen Serial Cardiac Readmissions and Death) trial. J Am Coll Cardiol 2009;55:53-60.

7. Pfisterer M, Buser P, Rickli H, et al. BNP-guided vs symptom-guided heart failure therapy: the Trial of Intensified vs Standard Medical Therapy in Elderly Patients With Congestive Heart Failure (TIME-CHF) randomized trial. JAMA 2009;301:383-92.

8. Eurlings LW, van Pol PE, Kok WE, et al. Management of chronic heart failure guided by individual N-terminal proB-type natriuretic peptide targets: results of the PRIMA (Can PRo-brain-natriuretic peptide guided therapy of chronic heart failure IMprove heart fAilure morbidity and mortality?) study. J Am Coll Cardiol 2010;56:2090-100.

9. Gaggin HK, Mohammed AA, Bhardwaj A, et al. Heart failure outcomes and benefits of NT-proBNP-guided management in the elderly: results from the prospective, randomized ProBNP outpatient tailored chronic heart failure therapy (PROTECT) study. J Card Fail 2012;18:626-34.

10. Troughton RW, Frampton CM, Yandle TG, et al. Treatment of heart failure guided by plasma aminoterminal brain natriuretic peptide (N-BNP) concentrations. Lancet 
2000;355:1126-30.

11. Persson H, Erntell H, Eriksson B, et al. Improved pharmacological therapy of chronic heart failure in primary care: a randomized Study of NT-proBNP Guided Management of Heart Failure--SIGNAL-HF (Swedish

Cite this article as: De Vecchis R. Natriuretic peptide-guided versus clinically guided therapy for chronic heart failure: careful expert clinical management can remove need for biomarkertailored dosing adjustment. J Thorac Dis 2017;9(11):4137-4140. doi: $10.21037 / j t d .2017 .10 .26$
Intervention study--Guidelines and NT-proBNP AnaLysis in Heart Failure). Eur J Heart Fail 2010;12:1300-8.

12. De Vecchis R, Esposito C, Cantatrione S. Natriuretic peptide-guided therapy: further research required for stillunresolved issues. Herz 2013;38:618-28. 